\title{
Breathing-Dependent Redox Activity in a Tetrathiafulvalene-Based Metal-Organic Framework
}

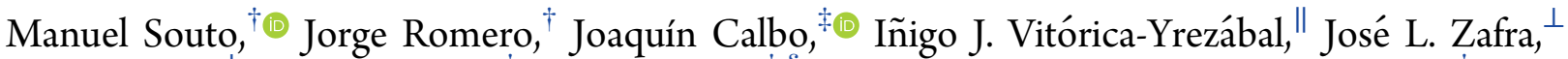 \\ Juan Casado, ${ }^{\perp \oplus}$ Enrique Ortí, ${ }^{\dagger}$ Aron Walsh, ${ }^{+, \delta \odot}$ and Guillermo Mínguez Espallargas, ${ }^{* \dagger}$ \\ ${ }^{\dagger}$ Instituto de Ciencia Molecular (ICMol), Universidad de Valencia, c/Catedrático José Beltrán, 2, 46980 Paterna, Spain \\ ${ }^{\ddagger}$ Department of Materials, Imperial College London, London SW7 2AZ, United Kingdom \\ ${ }^{\S}$ Department of Materials Science and Engineering, Yonsei University, Seoul 03722, Korea \\ "School of Chemistry, University of Manchester, Oxford Road, Manchester M13 9PL, United Kingdom \\ ${ }^{\perp}$ Departamento de Química Física, Universidad de Málaga, 29071 Málaga, Spain
}

Supporting Information

ABSTRACT: "Breathing" metal-organic frameworks (MOFs) that involve changes in their structural and physical properties upon an external stimulus are an interesting class of crystalline materials due to their range of potential applications including chemical sensors. The addition of redox activity opens up a new pathway for multifunctional "breathing" frameworks. Herein, we report the continuous breathing behavior of a tetrathiafulvalene (TTF)-based MOF, namely MUV-2, showing a reversible swelling (up to ca. $40 \%$ of the volume cell) upon solvent adsorption. Importantly, the planarity of the TTF linkers is influenced by the breathing behavior of the MOF, directly impacting on its electrochemical properties and thus opening the way for the development of new electrochemical sensors. Quantum chemical calculations and Raman spectroscopy have been used to provide insights into the tunability of the oxidation potential.

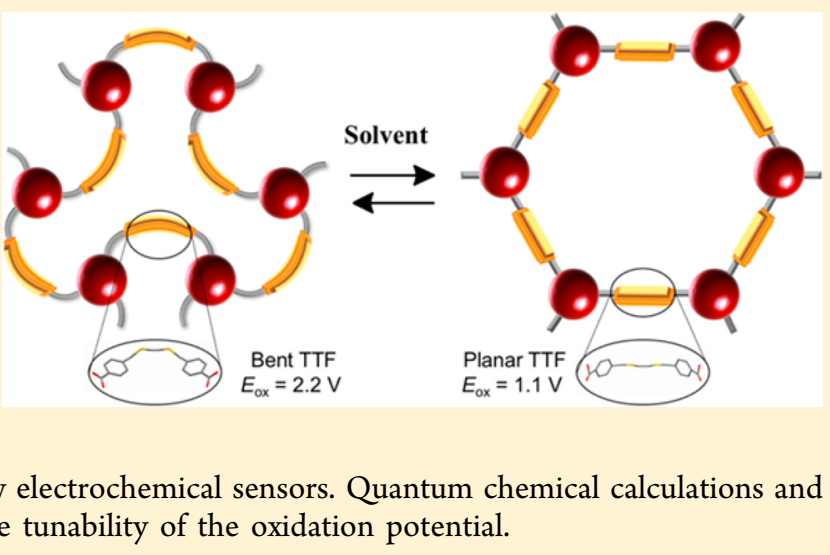

\section{INTRODUCTION}

Metal-organic frameworks (MOFs) are crystalline porous materials formed by inorganic building units and organic linkers that find several applications such as gas storage and separation, sensing, and catalysis. ${ }^{1}$ Although chemical makeup and robustness of MOFs have been key features in terms of possible industrial applications, during the past years there has been a growing interest in the design of flexible MOFs which undergo structural changes upon exposure to physical or chemical stimuli. $^{2-4}$ However, among the $\sim 70000$ MOFs reported to date, only $\sim 100$ exhibit flexible or "breathing" behavior in response to an external stimulus. ${ }^{3,5,6}$ Typically, the reversible transformations in flexible MOFs occur through well-defined phase transitions between wide-pore and narrowpore forms, as exemplified by the case of MIL-53. ${ }^{7,8}$ On the contrary, MOFs exhibiting large flexibility without well-defined transitions are extremely rare. ${ }^{5}$ This behavior is known as continuous breathing or "swelling", and the prototypical example of such behavior is the family of MIL-88., ${ }^{910}$ In both MIL-53 and MIL-88, the breathing behavior is driven by the rotation of the linkers with respect to the inorganic secondary building units (SBUs) based on a "kneecap" mechanism. However, there are not many examples in which the breathing motion is based on the flexibility of the ligand. $^{11,12}$
Breathing MOFs are even more interesting if they are able to tune the physical properties upon structural changes since they could be exploited for sensing or chemical-reporting applications. Recently, Farha and co-workers presented a Zrbased breathing MOF with flexible pyrene ligands where the linker conformational modifications are reflected in changes in its optical properties. ${ }^{12}$

On the other hand, redox-active $\mathrm{MOFs}^{13}$ have attracted a great deal of attention due to their potential application in electrocatalysis, ${ }^{14}$ electrochromic devices, ${ }^{15}$ conductors, ${ }^{16}$ and switches. ${ }^{17}$ In particular, tetrathiafulvalene (TTF)-based MOFs are promising systems for the development of multifunctional materials due to the intrinsically redox, electron-donor, and conducting properties of the TTF ligand. ${ }^{18-21}$ Along this line, Zhou and co-workers have demonstrated the reversible modulation of the breathing behavior of a TTF-based MOF upon oxidation and reduction of the TTF through modification of the linker's flexibility. ${ }^{22}$

Very recently, we have reported a highly stable and TTFbased MOF, namely MUV-2 (MUV: Materials of University of Valencia), which shows a hierarchical crystal structure with mesoporous one-dimensional channels and orthogonal micro-

Received: June 5, 2018

Published: July 24, 2018 
porous channels. ${ }^{23}$ This hierarchical MOF, based on 6connected $\left[\mathrm{Fe}_{3}\left(\mu_{3} \mathrm{O}\right)(\mathrm{COO})_{6}\right]$ SBUs and tetratopic tetrathiafulvalene tetrabenzoic $\left(\right.$ TTFTB $^{4-}$ ) ligands, shows high thermal and chemical stability, and has proven to be an efficient catalyst for the aerobic oxidation of dibenzothiophenes.

Herein, we report on the continuous breathing behavior of MUV-2 upon solvent adsorption by exploiting the flexibility of the boat-shaped TTFTB ligand (Figure 1). The reversible

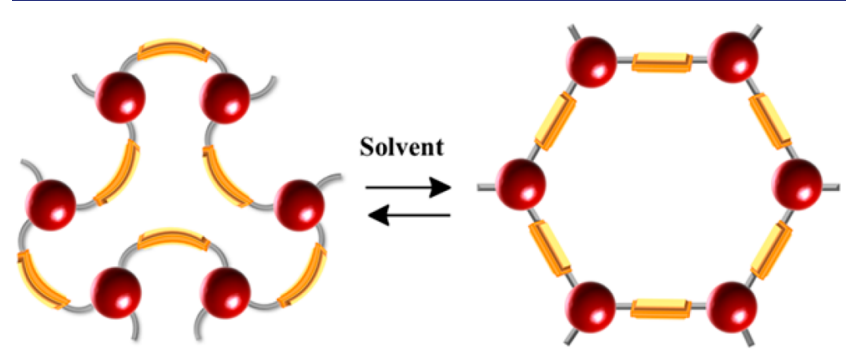

Figure 1. Schematic representation of the breathing behavior of MUV-2 upon solvent adsorption.

swelling of ca. $40 \%$ was studied in terms of cell dimensions between four different crystal structures of MUV-2 $(X)$, where $X=29,31,33$, and 35 , which were determined by single-crystal $\mathrm{X}$-ray diffraction as well as by the effect of different solvents on the framework by powder X-ray diffraction. Interestingly, the cell volume of the crystal structures is directly related to the degree of bending or planarity of the TTFTB ligands. Otherwise, the dihedral angles of the TTFTB linkers have a direct influence on the ionization energy of the molecules and, therefore, on the oxidation potential of MUV-2, as confirmed by quantum chemical calculations of the energy associated with electron removal. The tunability of the electrochemical properties of MUV-2 based on the breathing behavior has been demonstrated to have potential application as an electrochemical sensor. This is the first example of how the breathing behavior promoted by guest inclusion and based on a flexible ligand can modulate the redox properties of a MOF.

\section{RESULTS AND DISCUSSION}

2.1. X-ray Diffraction Analysis. MUV-2 was synthesized, washed, and characterized as previously described. ${ }^{23}$ Singlecrystal X-ray diffraction data were collected at the I19 beamline facilities at Diamond Light Source (UK) that were used to determine four different isolated phases of MUV-2 during the breathing motion (see the Supporting Information). As previously reported, ${ }^{23}$ the MUV-2 crystal structure is hexagonal (space group $P-62 m$ ) and, on the basis of the connection of six $\mathrm{Fe}_{3} \mathrm{O}(\mathrm{COO})_{6}$ SBUs and tetratopic TTFTB ligands, it can be simplified as a 3,6-connected network with ttp topology. ${ }^{24}$ This non-interpenetrated structure shows hexagonal mesoporous channels of ca. $3 \mathrm{~nm}$ in diameter along the $c$-axis direction formed by 6 TTFTB linkers and 6 SBUs (Figure 2). On the other hand, microporous channels of ca. $1 \mathrm{~nm}$ formed by 2 TTFTB ligands and 2 SBUs are disposed orthogonal to the mesoporous ones.

The breathing behavior of MUV-2 was studied in terms of four isolated crystal structures determined by single-crystal Xray diffraction using synchrotron radiation, denoted as MUV$2(29,31,33$, and 35) in accordance with the $a$ and $b$ unit cell parameters (Figure 3). The crystal structure of MUV-2 is contracted and expanded through the $a b$ plane, whereas the $c$
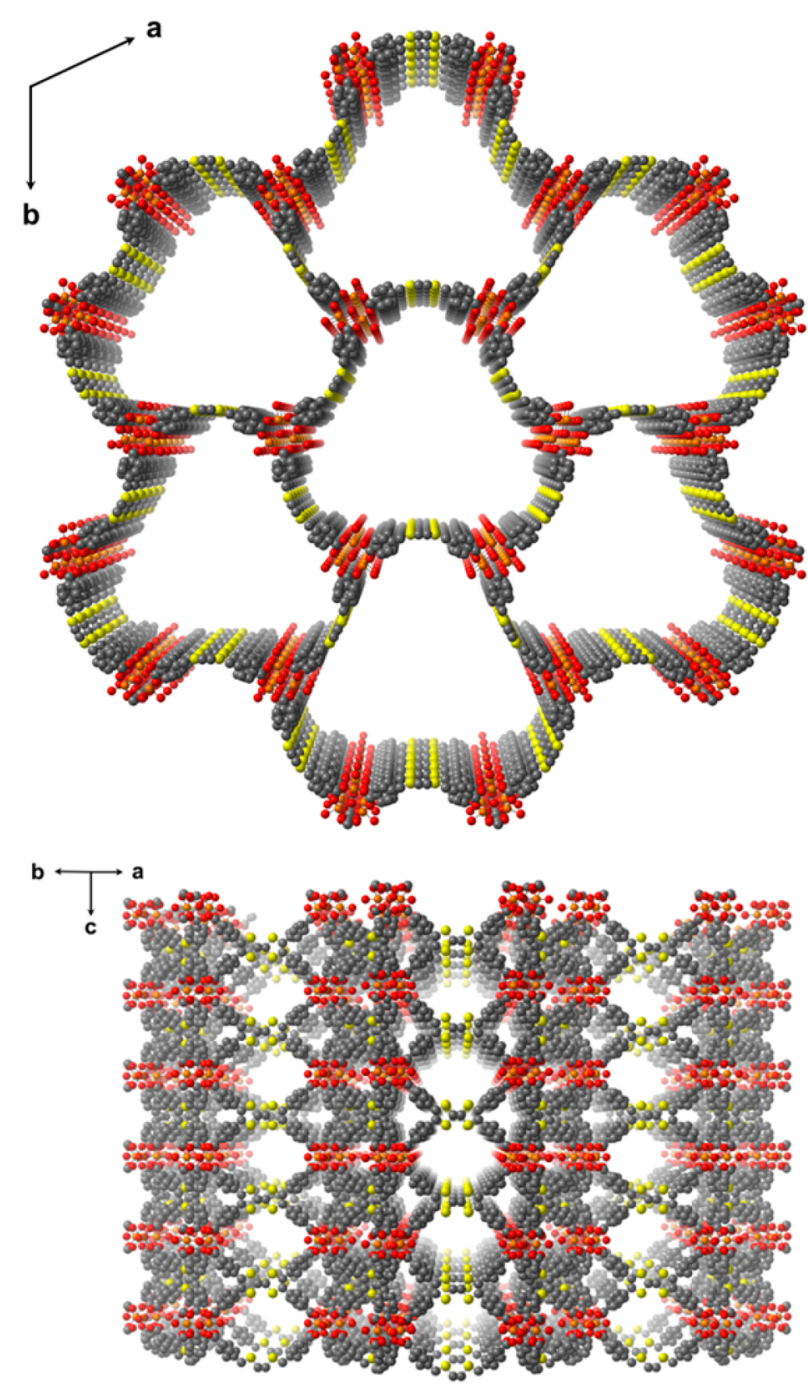

Figure 2. Overview of the crystal structure of MUV-2 showing the hexagonal mesoporous channels along the $c$-axis (top) and microporous channels orthogonal to the microporous ones (bottom). The gray, yellow, red, and orange balls represent the $\mathrm{C}, \mathrm{S}, \mathrm{O}$, and $\mathrm{Fe}$ atoms, respectively. For simplicity, hydrogens are omitted.

cell parameter remains almost unaltered, retaining in all four structures the same symmetry (hexagonal) and space group $(P$ $62 m$ ). The as-synthesized structure, MUV-2(33) (Figure 3c), shows a significant contraction upon drying treatment, providing the narrowest form, namely MUV-2(29) (Figure 3a). Upon adsorption of different solvents, the unit cell gradually increases, obtaining intermediate crystal structures such as MUV-2(31) (Figure 3b). The largest unit cell is observed using pyridine as guest, producing MUV-2(35) (Figure $3 \mathrm{~d}$ ), in which three pyridines molecules are coordinated to the $\mathrm{Fe}_{3} \mathrm{O}(\mathrm{COO})_{6}$ SBU replacing the three $\mathrm{H}_{2} \mathrm{O}$ molecules. The reversible continuous breathing behavior through the $a b$ plane is based on the flexibility of the TTFTB ligands, becoming much more planar upon solvent adsorption, and also on the partial rotation of the $\mathrm{Fe}_{3} \mathrm{O}(\mathrm{COO})_{6}$ SBUs. The shape of the pore changes from a triangle in MUV-2(29) to a hexagon in MUV-2(35), with a significant change of the distance between the calculated centroid of the $\mathrm{C}=\mathrm{C}$ central bond of adjacent TTFs (16.7 vs $29.5 \AA$, respectively) (Figure 3 ) and a total expansion of ca. $40 \%$ in the crystal structure volume (9427 vs $13163 \AA^{3}$ ). 
a)

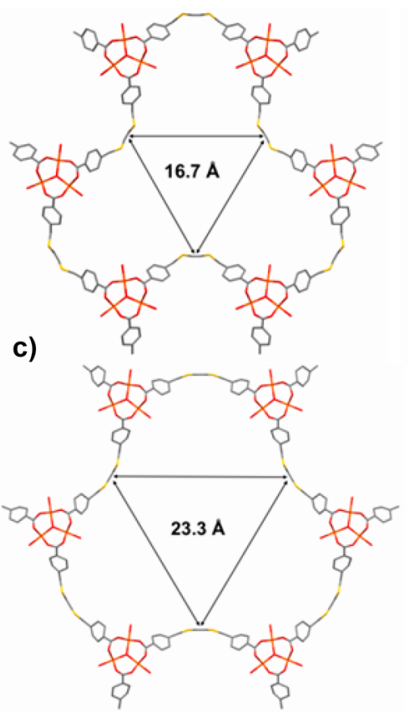

b)

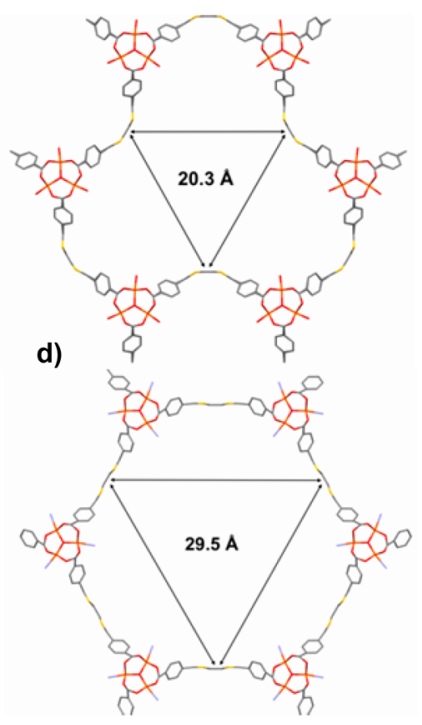

Figure 3. Crystal view of the pore opening evolution of (a) MUV2(29) $(a=b=29.4 \AA$; $c=12.5 \AA)$, (b) MUV-2(31) $(a=b=31.9 \AA$; $c=12.5 \AA)$, (c) MUV-2(33) $(a=b=33.3 \AA ; c=12.4 \AA)$, and (d) MUV-2(35) ( $a=b=35.4 \AA$; $c=12.1 \AA$ ) forms. The gray, yellow, red, orange, and blue ellipsoids represent the $\mathrm{C}, \mathrm{S}, \mathrm{O}, \mathrm{Fe}$, and $\mathrm{N}$ atoms, respectively. For simplicity, hydrogens are omitted.

According to the single-crystal structures, the reversible expansion and contraction of $\sim 40 \%$ between MUV-2(29) and MUV-2(35) occurs primarily through the $a b$ plane. This reversible expansion and contraction can also be observed using an optical microscope by exposing few desolvated single crystals of MUV-2(29) to pyridine solvent (Figure S2). The exposure of the single crystals to pyridine produces a reversible expansion between 20 and $30 \%$ of the width of the crystal, in agreement with the crystal indexing, which reveals that mesoporous channels are oriented along the length of the crystal (Figure S3). After evaporation of the pyridine solvent, the crystals recover the initial dimensions.

The evolution of the planarity of the TTFTB linker with the breathing behavior was analyzed by means of the dihedral angle $(\theta)$ formed by the two dithiole rings (planes $\mathrm{S} 1-\mathrm{C} 1-$ $\mathrm{C} 2-\mathrm{S} 2$ and $\mathrm{S} 3-\mathrm{C} 5-\mathrm{C} 6-\mathrm{S} 4)$ in the different crystal structures. The angles were found to be $69^{\circ}, 54^{\circ}, 41^{\circ}$, and $9^{\circ}$ for MUV-2(29), MUV-2(31), MUV-2(33), and MUV2(35), respectively (Figures 4 and S4). Moreover, the torsion

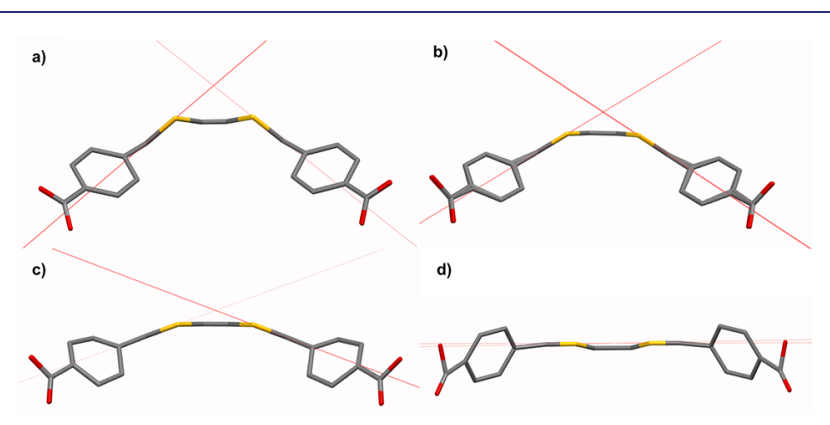

Figure 4. Partial views of the TTFTB ligand for the crystal structures of (a) MUV-2(29), (b) MUV-2(31), (c) MUV-2(33), and (d) MUV-2(35) highlighting the dihedral angles between the planes formed by the two dithiole rings $\left(\theta=69^{\circ}, 54^{\circ}, 41^{\circ}\right.$, and $9^{\circ}$, respectively).

angles of $\mathrm{S} 2-\mathrm{C} 3-\mathrm{S} 1-\mathrm{C} 1$, which are also related to the degree of planarity of the $\mathrm{TTF}^{25}$ decreased when the cell volume was increased, with values of $32^{\circ}, 26^{\circ}, 17^{\circ}$, and $14^{\circ}$, respectively.

2.2. Breathing Behavior upon Solvent Adsorption. The powder X-ray diffraction (PXRD) pattern of the activated material shows that the (100) peak appears at $2 \theta=3.45^{\circ}$ after solvent removal, in agreement with the contracted MUV-2(29) crystal structure. This peak shifts toward lower degrees after MUV-2(29) is soaked with different solvents during $24 \mathrm{~h}$ (Figure 5). As also observed in MIL-88, ${ }^{9,10}$ the breathing

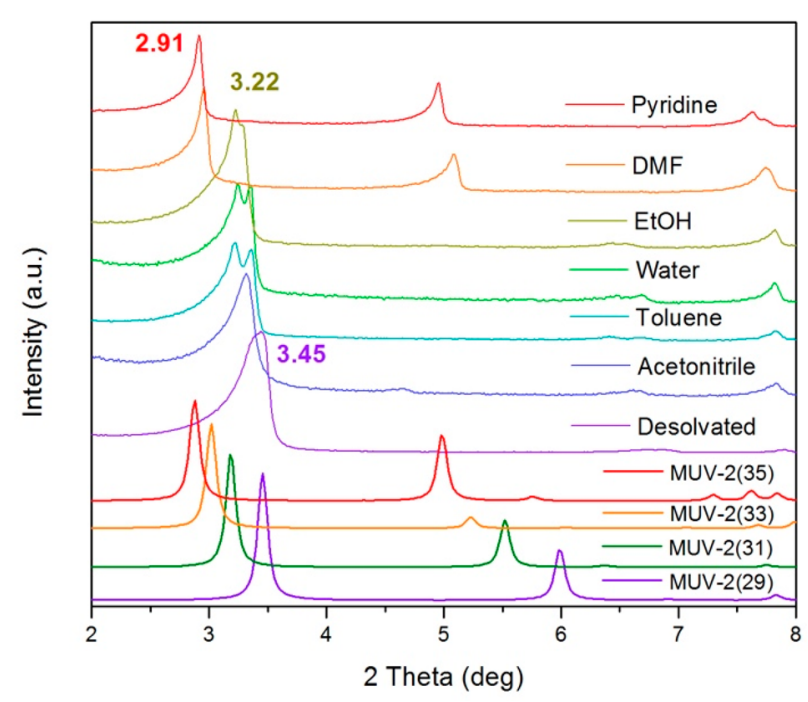

Figure 5. Evolution of PXRD patterns for MUV-2 after soaking with various solvents at room temperature. Simulated diffraction patterns of MUV-2(29), MUV-2(31), MUV-2(33), and MUV-2(35).

behavior is very selective, and the pore opening depends on the nature of the solvent. Strongly polar solvents such as pyridine, which is coordinated to the $\mathrm{Fe}_{3} \mathrm{O}(\mathrm{COO})_{6}$ SBU replacing the water molecules, generate the largest pore opening with a PXRD pattern similar to the one simulated from the crystal structure of MUV-2(35). Otherwise, exposure to ethanol, water, toluene, and acetonitrile yields intermediate cell variations with PXRD patterns similar to the ones simulated from MUV-2(33) and MUV-2(31) crystal structures, thus showing a continuous breathing behavior. Interestingly, PXRD patterns with ethanol, water, and toluene show the presence of two principal peaks, which can be related to a decrease of symmetry. The different breathing behavior of the solvents can be explained by the coordination of the polar organic solvent molecules with the inorganic $\mathrm{Fe}_{3} \mathrm{O}(\mathrm{COO})_{6}$ SBUs (as observed in the case of pyridine) or by weak interactions with the organic linkers, a behavior which is similar to that of the MIL88 family. ${ }^{9,10}$ Moreover, the reversible swelling of MUV-2(35) was confirmed by heating the soaked material with pyridine obtaining the initial desolvated PXRD pattern (Figure S5). It is important to note that the EPR spectra of MUV-2 in the different solvents do not show the presence of any signal related to $\mathrm{TTFTB}^{\bullet+}$ radical cation, indicating that the TTFTB moieties remain in the neutral state (Figure S6). Moreover, thermogravimetric analysis (TGA) of MUV-2 in different solvents was performed to determine the amounts of solvent molecules included in the pores in each case (Figure S7).

2.3. Quantum-Chemical Calculations. Based on the significant conformational changes of the TTF moiety 
observed in the different crystal structures, we aimed to analyze the impact of the TTF curvature on the electronic structure and redox properties of MUV-2. First, the minimum-energy structure of MUV-2 was obtained upon full ion and lattice relaxation under the density functional theory framework. The range-separated hybrid exchange-correlation HSE06 functional was employed along with the "Tier 1 " numerical basis set using the FHI-AIMS package ${ }^{26-28}$ (see the SI for full computational details). A high-spin configuration for the $\mathrm{Fe}$ atoms was adopted in line with the experimental evidence. ${ }^{23}$ The optimized structure of MUV-2 (Figure S8) compares well with the X-ray structure of as-synthetized MUV-2(33) and provides optimized lattice parameters of $a=34.03 \AA$, $b=34.06$ $\AA, c=12.15 \AA, \alpha=90.4^{\circ}, \beta=89.5^{\circ}, \gamma=120.2^{\circ}$, a TTF boat dihedral angle of $\theta=46.24^{\circ}$, and a pore opening (distance between centroid of the $\mathrm{C}=\mathrm{C}$ central bond of adjacent TTFs) of $23.95 \AA$. Note that substitution of the two coordinating water molecules by acetonitrile or pyridine barely affects the TTF tilting, with a boat dihedral angle $\theta$ of 44.82 and $45.53^{\circ}$, respectively (Figure S9). The breathing behavior of MUV-2 must therefore be originated from long-range electrostatic effects (solvent polarity) and/or medium-range electron correlation effects (noncovalent interactions). ${ }^{10}$

Analysis of the electronic density of states (DoS) indicates a small bandgap of $0.86 \mathrm{eV}$ originated in the $\beta$-spin manifold (Figure 6a). Projected DoS (PDoS) allows unequivocal
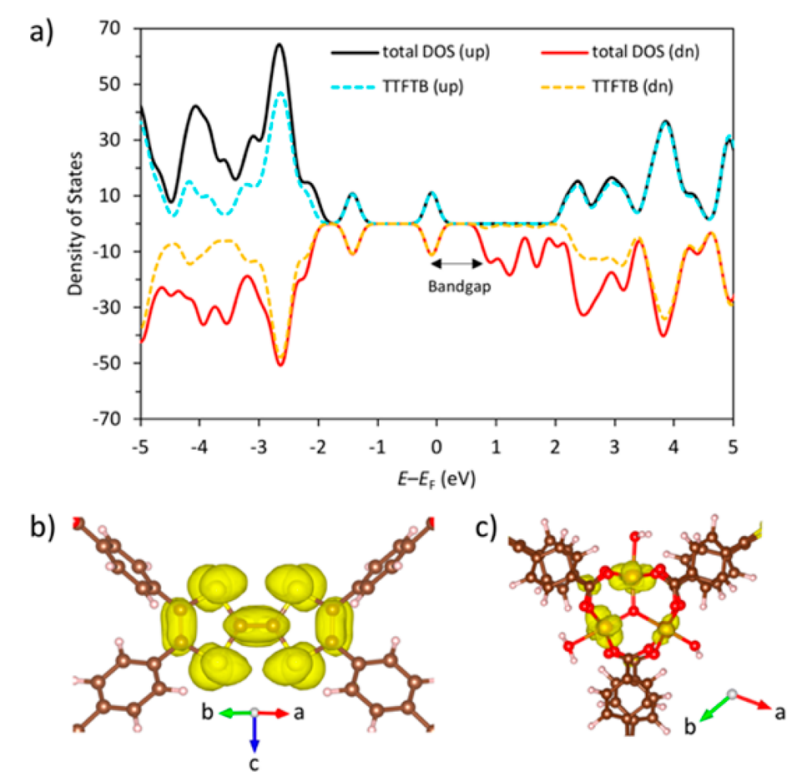

Figure 6. (a) Electronic density of states (DoS) calculated at the DFT-HSE06 level of theory for the optimized geometry of MUV-2. The highest occupied state $\left(E_{\mathrm{F}}\right)$ is set to $0 \mathrm{eV}$. Spin-up (up) and spindown $(\mathrm{dn})$ are differentiated with opposite signs. The contribution of the ligand atoms to the DoS is included. (b, c) Eigenstate densities (isovalue $=0.001$ ) for the HOCO and LUCO, respectively, of the $\beta$ spin channel.

assignment of the frontier crystal orbitals (FCOs): the highest occupied crystal orbital (HOCO) belongs to the organic TTF ligand, whereas the lowest unoccupied crystal orbital (LUCO) is localized in the inorganic cluster (Figures 6a and S10). Visualization of the electronic eigenstate densities confirmed the nature of the FCOs with a HOCO (Figure 6b) analogous to the highest occupied molecular orbital of TTF (Figure S11) and a $d$-orbital HUCO localized on the Fe atoms (Figure 6c).
These results suggest that reduction of the MOF must occur in the inorganic cluster, whereas oxidation processes will be centered in the TTF-based ligand. The spin density of the MUV-2 oxidized by one-electron per TTFTB ligand (unit cell with three positive charges) confirmed the electron density extraction from the TTF moiety upon oxidation (Figure S12).

To analyze the impact of the breathing motion into the electrochemical properties of MUV-2, experimental X-ray crystal structures were further employed to achieve geometries with variable TTF curvature (see the SI). The schematic representation of the FCOs versus the characteristic TTF boat dihedral angle $\theta$ shows a significant stabilization of $0.41 \mathrm{eV}$ for the TTF-centered HOCO going from $\theta=30.87^{\circ}$ to $66.11^{\circ}$ (Figure 7). This stabilization has already been reported in

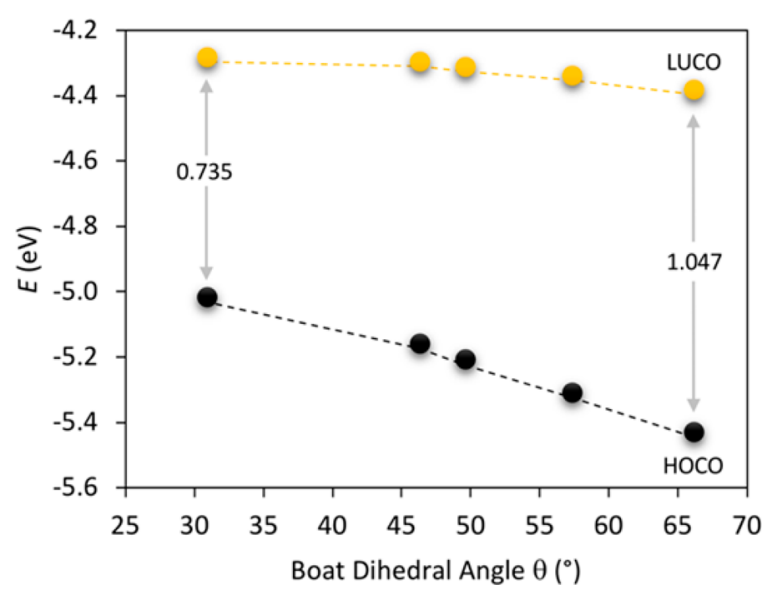

Figure 7. One-electron energy diagram of the frontier crystal orbitals of MUV-2 with different values for the TTF boat dihedral angle $\theta$ as defined in Figure 4. The electronic bandgap (in $\mathrm{eV}$ ) for the extreme cases is indicated. Dashed lines are included to guide the eye.

molecular systems containing the TTF unit, and is ascribed to an inefficient $6 \pi$-electron delocalization that disrupts its donor ability upon deviation from planarity. ${ }^{29-31}$ Otherwise, the Fecentered LUCO remains practically unaffected upon TTF bending, with an energy range of $[-4.30,-4.40] \mathrm{eV}$. As a result, an increase of $>0.3 \mathrm{eV}$ in the electronic bandgap is predicted going from MOF structures with a planar TTF to those with a highly curved ligand (Figure 7).

We took advantage of the localized nature of the HOCO into the $\pi$-conjugated TTF core to analyze the electrochemical properties of MUV-2 upon ligand bending by performing highlevel molecular calculations. The minimum-energy structure of the TTFTB moiety in its neutral (fully protonated) form was obtained at the DFT-PBE0/cc-pVDZ level of theory in the gas phase (Figure S11). Starting from this geometry, a relaxed scan was performed at the same level by varying the characteristic TTF boat dihedral angle. Theoretical calculations predict a small energy penalty upon boat bending, with a large conformational space $0^{\circ}<\theta<80^{\circ}$ accessible within $5 \mathrm{kcal} /$ mol (Figure S17). Initial analysis of the molecular orbitals indicates a significant stabilization of the TTF-centered highest occupied molecular orbital (HOMO) upon bending (from $-4.93 \mathrm{eV}$ at $\theta=4.84^{\circ}$ to $-5.69 \mathrm{eV}$ at $\theta=87.34^{\circ}$; Table S4), in good accord with the solid-state calculations (Figure 7 ).

The first ionization potential (IP) of the TTFTB ligand was then calculated along the relaxed scan to estimate the effect of the breathing motion into the oxidation potential of MUV-2 
(see the SI). As shown in Figure 8, the IP of TTFTB monotonically decreases upon planarization $(6.60 \mathrm{eV}$ at $\theta=$

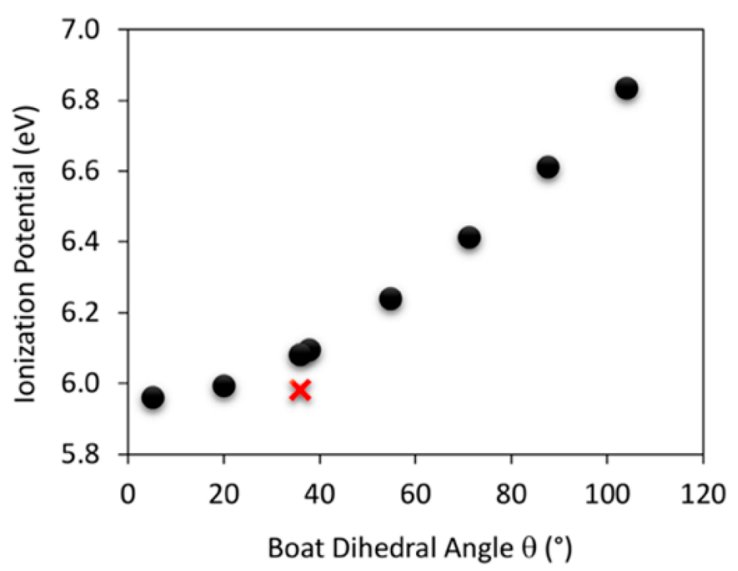

Figure 8. Ionization potential (IP) calculated for the TTFTB ligand molecule from total energy differences at the DFT-PBE0/cc-pVDZ level of theory as a function of the characteristic TTF boat dihedral angle $\theta$. The adiabatic IP calculated for TTFTB as shown as a red cross.

$87.34^{\circ}$ vs $6.08 \mathrm{eV}$ at $\left.\theta=37.61^{\circ}\right)$. It is well-known that the TTF unit planarizes upon one-electron oxidation. ${ }^{32,33}$ However, the effect in IP due to the strained rigidity of the ligand in MUV-2 is calculated to be only of $0.10 \mathrm{eV}$ at the minimum-energy boat dihedral angle $\theta=35.72^{\circ}$ (adiabatic IP $=5.97 \mathrm{eV}$ ). Further planarization of the ligand leads to a small decrease of IP, with the lowest value of $5.95 \mathrm{eV}$ at $\theta=4.84^{\circ}$. Theoretical calculations therefore predict a significant change in the electrochemical properties of MUV-2 upon swelling, with a systematic increase in ease of oxidation upon planarization of the TTFTB ligand.

2.4. Electrochemical Properties. Encouraged by the theoretical results demonstrating the influence of the breathing motion on the electrochemical properties of MUV-2, solidstate cyclic voltammetry (CV) and differential pulse voltammetry (DPV) of MUV-2 were performed in different solvents (Figure 9). We have previously reported the $\mathrm{CV}$ of MUV-2 in $\mathrm{CH}_{3} \mathrm{CN}$ up to $1.6 \mathrm{~V}$ (vs $\mathrm{Ag} / \mathrm{AgCl}$ ), which showed a reversible redox peak attributed to the reduction process of $\mathrm{Fe}^{3+} / \mathrm{Fe}^{2+}$ around $-0.9 \mathrm{~V}$, but no peak related to the oxidation of the TTFTB linkers was observed. ${ }^{23}$ Upon increasing the potential up to $3 \mathrm{~V}$, the CV of MUV-2 shows an irreversible redox peak at $2.2 \mathrm{~V}$, which can be attributed to the oxidation process of the TTFTB units (Figures 9 and S20). To investigate the relationship between the $\mathrm{Fe}$ and TTFTB moieties, the Randles-Sevcik equation ${ }^{34}$ was used to determine the Fe/TTFTB molar ratio, which was found to have a value of 1.8. This value is close to the formula of MUV2 (two $\mathrm{Fe}_{3} \mathrm{O}$ SBUs per three TTFTB linkers).

The solid-state CV of MUV-2 in pyridine shows that the formal redox potential assigned to the oxidation of TTFTB significantly decreases, and two irreversible redox waves were observed at 1.02 and $1.16 \mathrm{~V}$ attributed to the two oxidation processes of TTFTB to TTFTB $^{\bullet+}$ and TTFTB $^{2+}$, respectively (Figures 9 and S20). These oxidation potentials are closer to those reported for other TTF-based MOFs. ${ }^{19}$ The decrease of the oxidation potential of MUV-2 when using pyridine as solvent can be explained by the increased planarity of the TTFTB linkers, which significantly decreased the ionization

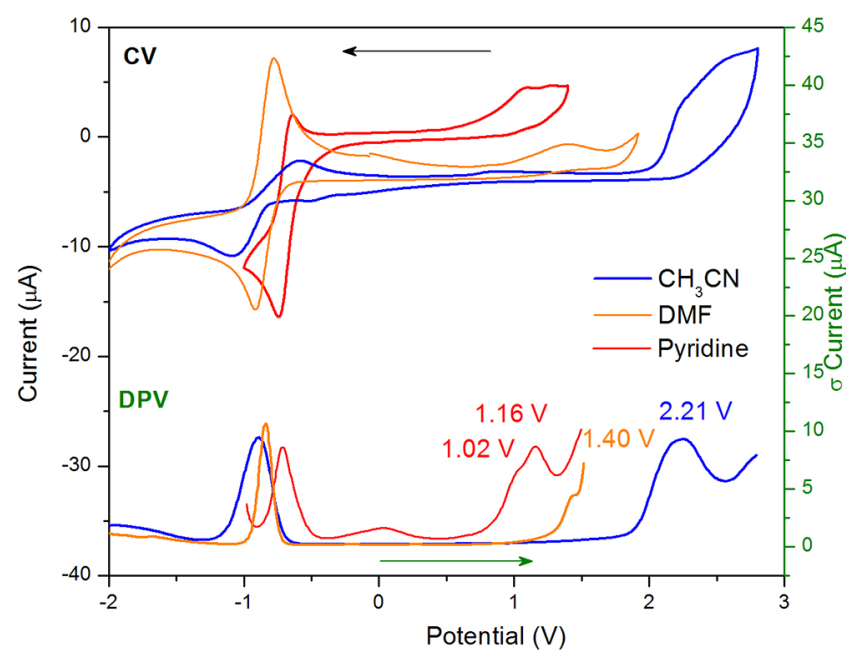

Figure 9. Solid-state cyclic voltammetry (CV) and differential pulse voltammetry (DPV) of MUV-2 in $\mathrm{CH}_{3} \mathrm{CN}$ (blue line), DMF (orange line), and pyridine (red line) using $\mathrm{TBAPF}_{6} 0.1 \mathrm{M}$ as electrolyte and $0.1 \mathrm{~V} / \mathrm{s}$ scan rate. Platinum wire was used as the counter electrode and silver wire as the pseudoreference electrode. Ferrocene was added as internal standard. All potentials are reported versus $\mathrm{Ag} / \mathrm{AgCl}$.

potential as demonstrated by the theoretical calculations. Interestingly, when using a solvent such as DMF, which produces an intermediate degree of swelling and planarity of the TTFTB ligands, the formal oxidation potential is slightly higher than that in pyridine $(1.40 \mathrm{~V})$ (Figures 9 and S21). Hence, the electrochemical properties of MUV-2 can be modulated by the solvent-induced breathing behavior.

Based on the idea that the electrochemical properties can be tuned by the effect of the different solvents on the breathing behavior, DPV measurements of MUV-2 in $\mathrm{CH}_{3} \mathrm{CN}$ were performed in the range of $0-2.4 \mathrm{~V}$ after addition of consecutive aliquots of pyridine (Figure 10). The oxidation peak which initially appears at $2.2 \mathrm{~V}$ gradually shifts toward lower potentials when pyridine is added, in agreement with the lower ionization potential of MUV-2 when it is soaked with pyridine due to planarization of the TTFTB linker. After

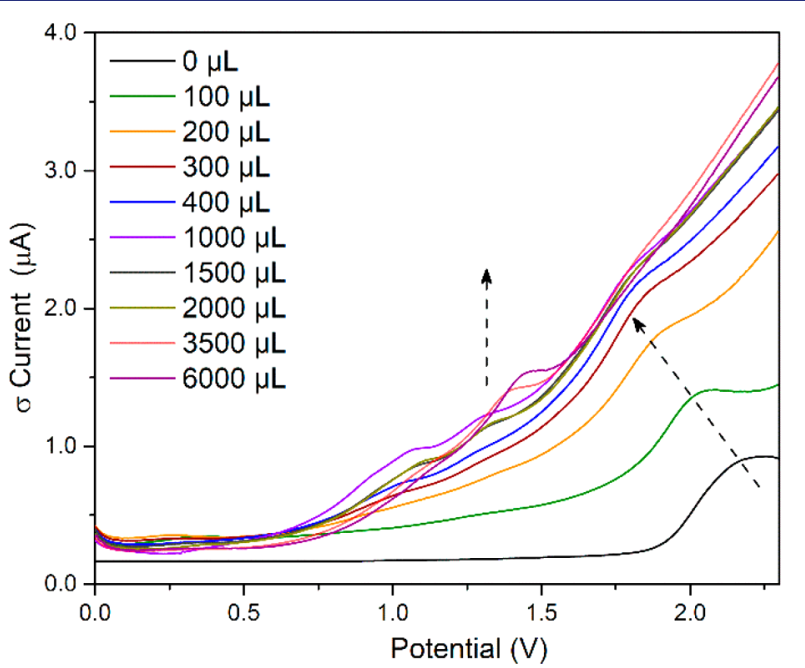

Figure 10. Differential pulse voltammetry (DPV) of MUV-2 in $\mathrm{CH}_{3} \mathrm{CN}$ after consecutive addition of aliquots of pyridine using $\mathrm{TBAPF}_{6} 0.1 \mathrm{M}$ as electrolyte. Platinum wire was used as the counter electrode and silver wire as the pseudoreference electrode. 
addition of $100 \mu \mathrm{L}(1 \% \mathrm{v} / \mathrm{v})$ and $300 \mu \mathrm{L}(3 \% \mathrm{v} / \mathrm{v})$ of pyridine, the oxidation potential moves to 2.0 and $1.8 \mathrm{~V}$, respectively. Moreover, we also observe the appearance of new peaks in the range of 1.0-1.5 V, which are close to the oxidation peak of MUV-2 using exclusively pyridine as solvent (Figure 10). The presence of numerous peaks indicates different degrees of breathing behavior probably due to diffusion effects. In addition, $\mathrm{CV}$ measurements performing the same procedure (Figure S23) also show a gradual shift of the oxidation peak toward lower potential. Thus, consecutive addition of pyridine induces the expansion toward MUV-2(35) form and the consequent planarization of TTFTB linkers producing significant changes in its electrochemical response.

Motivated by the idea of isolating the oxidized MOF (oxMUV-2) to study the pore opening of the system, we oxidized MUV-2 deposited on the electrode applying a constant potential (1.8 V using DMF as solvent, or $1.1 \mathrm{~V}$ using pyridine as solvent). EPR spectra demonstrate that the TTFTB linkers were oxidized to $\mathrm{TTFTB}^{\bullet+}$ in agreement with the presence of the typical signal for the radical cation species centered at $g=$ 2.006 (Figure S25). However, the PXRD pattern shows that crystallinity is decreased (Figure S26), and IR spectra suggest that some of the TTFTB linkers are uncoordinated to the metal due to the presence of bands at $1691 \mathrm{~cm}^{-1}$ assigned to free $\mathrm{C}=\mathrm{O}$ groups (Figure S27). This suggests that an irreversible decarboxylation upon oxidation is produced related to the Kolbe reaction, as it has been observed for other TTFbearing carboxylic moieties. ${ }^{35,36}$ Some electrochemical studies performed on different TTF-bearing carboxylic acids suggest that a decarboxylation process occurs only during the second oxidation step (the formation of the dication species), which is irreversible. $^{37}$ In the case of MUV-2, it is not possible to control the oxidation up to the radical cation state since the two oxidation peaks are very close.

2.5. Raman Spectroscopy. The vibrational fingerprint of MUV-2 can provide structural information on the dynamics and of the structural changes in the interior of the pores under a variety of conditions. ${ }^{38,39}$ Hence, a complete study of the vibrational Raman properties of desolvated MUV-2, upon solvation with pyridine, acetonitrile, and DMF (py-MUV-2, ACN-MUV-2, and DMF-MUV-2) and electrochemically oxidized (ox-MUV-2) has been carried out with two different Raman laser excitation lines (633 and 785 nm, shown in Figure 11 and Figure S28, respectively).

The 785 and $633 \mathrm{~nm}$ Raman spectra of the desolvated sample are dominated by the presence of three main bands in the $1650-1400 \mathrm{~cm}^{-1}$ interval: the band at $1607 \mathrm{~cm}^{-1}$ (i.e., due to the $\mathrm{C}=\mathrm{C}$ stretching vibration of the benzene moieties), the band at $1540 \mathrm{~cm}^{-1}$ (i.e., assigned to the $\mathrm{C}=\mathrm{C}$ stretching mode of the TTF moiety), and the broad band at $1413 \mathrm{~cm}^{-1}$ (i.e., arising from the stretching modes of the carboxylate groups). See Figures S18 and S19 for the simulated Raman spectra. Importantly, the relative Raman intensity pattern of these bands consists of the strongest band at $1413 \mathrm{~cm}^{-1}$ followed by the band at $1607 \mathrm{~cm}^{-1}$ with medium intensity. In the case of the electrochemically oxidized ox-MUV-2, the spectral intensity pattern in the $1650-1400 \mathrm{~cm}^{-1}$ interval is reversed compared with the unoxidized material, with the strongest feature now at $1607 \mathrm{~cm}^{-1}$ and those at $1440-1400 \mathrm{~cm}^{-1}$ as medium bands. In addition, two new weak bands appear at 1501 and $1438 \mathrm{~cm}^{-1}$, which are assigned to those Raman modes in the oxidized TTF moiety that result from the

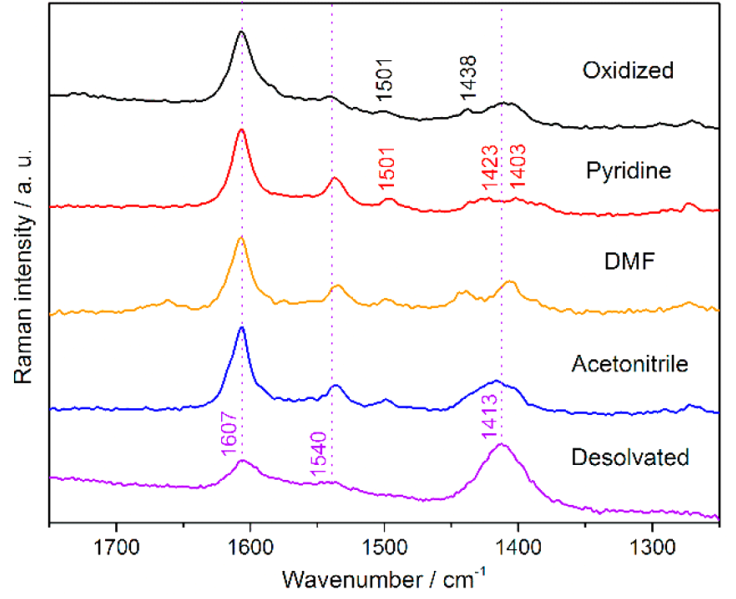

Figure 11. Raman spectra $(633 \mathrm{~nm})$ of desolvated MUV-2 (purple line) and solvated with acetonitrile (blue line), DMF (orange line), and pyridine (red line) and electrochemically oxidized ox-MUV-2 (black line) at $298 \mathrm{~K}$ in solid state. See Figure S29 for a larger spectral range.

frequency softening of the $\mathrm{C}=\mathrm{C}$ stretching modes upon oxidation of the TTF unit.

Solvation causes structural changes that make their Raman spectra resemble that of the oxidized material. This is inferred by the relative intensity pattern of the bands at 1607 (strongest) and $1440-1400 \mathrm{~cm}^{-1}$ (medium), and by the appearance of new bands at 1501 and $1440-1410 \mathrm{~cm}^{-1}$ in the solvated samples in analogy with the Raman spectrum for oxMUV-2. Since oxidation of TTF produces its backbone planarization, the spectral similitude with the solvated materials indicates that solvation also produces the planarization of the TTF unit of MUV-2. Looking more in detail to the $1440-1400 \mathrm{~cm}^{-1}$ region of the solvated samples, significant differences are found between the spectra. Whereas four peaks are observed for py-MUV-2, a main band with two shoulders is resolved in ACN-MUV-2 (for DMF-MUV-2 the solvent bands prevent further comparisons). It seems that the spectral changes in this region are less marked for ACN-MUV-2 than for py-MUV-2 (one peak in the former and four peaks in the latter), revealing that adsorption of acetonitrile induces smaller changes in the Raman spectra. This is in perfect agreement with the structural features obtained by $\mathrm{X}$-ray diffraction.

\section{CONCLUSIONS}

In summary, we have reported the continuous breathing behavior of a TTF-based MOF, namely MUV-2, which shows up to $40 \%$ of a reversible expansion upon solvent adsorption. Interestingly, the planarity of the TTF linkers is influenced by the breathing behavior as demonstrated by single-crystal X-ray diffraction and Raman spectroscopy. Theoretical calculations show that the degree of planarity of the TTF ligands has a direct influence on the electrochemical properties and the tunability of the oxidation potential of MUV-2 upon adsorption of solvents. These results open the way to develop new electrochemical sensors based on the breathing behavior and new materials with pore opening control upon application of electrochemical potential. 


\section{ASSOCIATED CONTENT}

\section{S Supporting Information}

The Supporting Information is available free of charge on the ACS Publications website at DOI: 10.1021/jacs.8b05890.

General methods and materials, theoretical calculations, electrochemical measurements, characterization of oxidized MUV-2, Raman spectroscopy (PDF)

X-ray data for MUV-2(29) (CIF)

X-ray data for MUV-2(31) (CIF)

X-ray data for MUV-2(33) (CIF)

X-ray data for MUV-2(35) (CIF)

\section{AUTHOR INFORMATION}

\section{Corresponding Author}

*guillermo.minguez@uv.es

\section{ORCID $\odot$}

Manuel Souto: 0000-0003-3491-6984 Joaquín Calbo: 0000-0003-4729-0757 Juan Casado: 0000-0003-0373-1303 Enrique Ortí: 0000-0001-9544-8286 Aron Walsh: 0000-0001-5460-7033 Guillermo Mínguez Espallargas: 0000-0001-7855-1003 Notes

The authors declare no competing financial interest.

\section{ACKNOWLEDGMENTS}

The work has been supported by the European Union (ERC2016-CoG 724681-S-CAGE) and the Spanish MINECO (CTQ2014-59209-P, CTQ2017-89528-P, and CTQ201569391-P). We also thank the Spanish government for the provision of a Maria de Maeztu project (MDM-2015-0538). G.M.E. and M.S. thank MINECO for a Ramón y Cajal and a Juan de la Cierva-Formación fellowship, respectively. We thank the Diamond Light Source (UK) for the synchrotron beamtime. The work at ICL has been supported by the Leverhulme Trust and the Royal Society. Through our membership in the UK's HEC Materials Chemistry Consortium, which is funded by EPSRC (EP/L000202), this work used the ARCHER supercomputing service.

\section{REFERENCES}

(1) Maurin, G.; Serre, C.; Cooper, A.; Férey, G. Chem. Soc. Rev. 2017, 46, 3104.

(2) Férey, G.; Serre, C. Chem. Soc. Rev. 2009, 38, 1380.

(3) Schneemann, A.; Bon, V.; Schwedler, I.; Senkovska, I.; Kaskel, S.; Fischer, R. A. Chem. Soc. Rev. 2014, 43, 6062.

(4) Morris, R. E.; Brammer, L. Chem. Soc. Rev. 2017, 46, 5444.

(5) Carrington, E. J.; McAnally, C. A.; Fletcher, A. J.; Thompson, S. P.; Warren, M.; Brammer, L. Nat. Chem. 2017, 9, 882.

(6) Moghadam, P. Z.; Li, A.; Wiggin, S. B.; Tao, A.; Maloney, A. G. P.; Wood, P. A.; Ward, S. C.; Fairen-Jimenez, D. Chem. Mater. 2017, 29, 2618.

(7) Serre, C.; Millange, F.; Thouvenot, C.; Noguès, M.; Marsolier, G.; Louër, D.; Férey, G. J. Am. Chem. Soc. 2002, 124, 13519.

(8) Horcajada, P.; Serre, C.; Maurin, G.; Ramsahye, N. A.; Balas, F.; Sebban, M.; Taulelle, F.; Vallet-Regi, M.; Ferey, G. J. Am. Chem. Soc. 2008, 130, 6774.

(9) Mellot-Draznieks, C.; Serre, C.; Surblé, S.; Audebrand, N.; Férey, G. J. Am. Chem. Soc. 2005, 127, 16273.

(10) Serre, C.; Mellot-Draznieks, C.; Surblé, S.; Audebrand, N.; Filinchuk, Y.; Férey, G. Science 2007, 315, 1828.

(11) Lin, Z.-J.; Lü, J.; Hong, M.; Cao, R. Chem. Soc. Rev. 2014, 43, 5867.
(12) Deria, P.; Gómez-Gualdrón, D. A.; Bury, W.; Schaef, H. T.; Wang, T. C.; Thallapally, P. K.; Sarjeant, A. A.; Snurr, R. Q.; Hupp, J. T.; Farha, O. K. J. Am. Chem. Soc. 2015, 137, 13183.

(13) D’Alessandro, D. M. Chem. Commun. 2016, 52, 8957.

(14) Ahrenholtz, S. R.; Epley, C. C.; Morris, A. J. J. Am. Chem. Soc. 2014, 136, 2464.

(15) Kung, C. W.; Wang, T. C.; Mondloch, J. E.; Fairen-Jimenez, D.; Gardner, D. M.; Bury, W.; Klingsporn, J. M.; Barnes, J. C.; Van Duyne, R.; Stoddart, J. F.; Wasielewski, M. R.; Farha, O. K.; Hupp, J. T. Chem. Mater. 2013, 25, 5012.

(16) Darago, L. E.; Aubrey, M. L.; Yu, C. J.; Gonzalez, M. I.; Long, J. R. J. Am. Chem. Soc. 2015, 137, 15703.

(17) Gui, B.; Meng, X.; Chen, Y.; Tian, J.; Liu, G.; Shen, C.; Zeller, M.; Yuan, D.; Wang, C. Chem. Mater. 2015, 27, 6426.

(18) Wang, H.-Y.; Cui, L.; Xie, J.-Z.; Leong, C. F.; D’Alessandro, D. M.; Zuo, J.-L. Coord. Chem. Rev. 2017, 345, 342.

(19) Wang, H.-Y.; Ge, J.-Y.; Hua, C.; Jiao, C.-Q.; Wu, Y.; Leong, C. F.; D'Alessandro, D. M.; Liu, T.; Zuo, J.-L. Angew. Chem., Int. Ed. 2017, 56, 5465.

(20) Narayan, T. C.; Miyakai, T.; Seki, S.; Dincă, M. J. Am. Chem. Soc. 2012, 134, 12932.

(21) Park, S. S.; Hontz, E. R.; Sun, L.; Hendon, C. H.; Walsh, A.; Van Voorhis, T.; Dincă, M. J. Am. Chem. Soc. 2015, 137, 1774.

(22) Su, J.; Yuan, S.; Wang, H.-Y.; Huang, L.; Ge, J.-Y.; Joseph, E.; Qin, J.; Cagin, T.; Zuo, J.-L.; Zhou, H.-C. Nat. Commun. 2017, 8, 2008.

(23) Souto, M.; Santiago-Portillo, A.; Palomino, M.; VitoricaYrzebal, I.; Vieira, B. J. C.; Waerenborgh, J. C. C.; Valencia, S.; Navalon, S.; Rey, F.; Garcia, H.; Minguez Espallargas, G. Chem. Sci. 2018, 9, 2413.

(24) Although the topology of MUV-2 has been previously described as a binoidal $(4,6)$-c net, ${ }^{23}$ the correct topology is a binoidal $(3,6)$-c net since the linker should be abstracted as two threecoordinated nodes.

(25) Mukherjee, V.; Singh, N. P. Spectrochim. Acta, Part A 2014, 117, 315.

(26) Blum, V.; Gehrke, R.; Hanke, F.; Havu, P.; Havu, V.; Ren, X.; Reuter, K.; Scheffler, M. Comput. Phys. Commun. 2009, 180, 2175.

(27) Havu, V.; Blum, V.; Havu, P.; Scheffler, M. J. Comput. Phys. 2009, 228, 8367.

(28) Ren, X.; Rinke, P.; Blum, V.; Wieferink, J.; Tkatchenko, A.; Sanfilippo, A.; Reuter, K.; Scheffler, M. New J. Phys. 2012, 14, 53020.

(29) Hansen, T. K.; Joergensen, T.; Jensen, F.; Thygesen, P. H.; Christiansen, K.; Hursthouse, M. B.; Harman, M. E.; Malik, M. A.; Girmay, B. J. Org. Chem. 1993, 58, 1359.

(30) Wang, C. S.; Bryce, M. R.; Batsanov, A. S.; Howard, J. A. K. Chem. - Eur. J. 1997, 3, 1679.

(31) Berridge, R.; Serebryakov, I. M.; Skabara, P. J.; Ortí, E.; Viruela, R.; Pou-Amérigo, R.; Coles, S. J.; Hursthouse, M. B. J. Mater. Chem. 2004, 14, 2822

(32) Adamo, C.; Arnaud, R.; Scalmani, G.; Müller, H.; Sahli, F.; Barone, V. J. Phys. Chem. B 1999, 103, 6863.

(33) Yakushi, K.; Nishimura, S.; Sugano, T.; Kuroda, H.; Ikemoto, I. Acta Crystallogr., Sect. B: Struct. Crystallogr. Cryst. Chem. 1980, 36, 358.

(34) Zhang, T.; Hu, Y. Q.; Han, T.; Zhai, Y. Q.; Zheng, Y. Z. ACS Appl. Mater. Interfaces 2018, 10, 15786.

(35) Nguyen, T. L. A.; Demir-Cakan, R.; Devic, T.; Morcrette, M.; Ahnfeldt, T.; Auban-Senzier, P.; Stock, N.; Goncalves, A. M.; Filinchuk, Y.; Tarascon, J. M.; Férey, G. Inorg. Chem. 2010, 49, 7135.

(36) Nguyen, T. L. A.; Devic, T.; Mialane, P.; Rivière, E.; Sonnauer, A.; Stock, N.; Demir-Cakan, R.; Morcrette, M.; Livage, C.; Marrot, J.; Tarascon, J. M.; Férey, G. Inorg. Chem. 2010, 49, 10710.

(37) Idriss, K. A.; Chambers, J. Q. J. J. Electroanal. Chem. 1980, 109, 341 .

(38) Krylov, A.; Vtyurin, A.; Petkov, P.; Senkovska, I.; Maliuta, M.; Bon, V.; Heine, T.; Kaskel, S.; Slyusareva, E. Phys. Chem. Chem. Phys. 2017, 19, 32099. 
(39) Hoffman, A. E. J.; Vanduyfhuys, L.; Nevjestić, I.; Wieme, J.; Rogge, S. M. J.; Depauw, H.; Van Der Voort, P.; Vrielinck, H.; Van Speybroeck, V. J. Phys. Chem. C 2018, 122, 2734. 